Check for updates

Cite this: Mater. Adv., 2020, 1, 1739

Received 24th March 2020, Accepted 9th July 2020

DOI: 10.1039/d0ma00124d

rsc.li/materials-advances

\title{
A rational design of a cancer-specific and lysosome-targeted fluorescence nanoprobe for glutathione imaging in living cells $\dagger$
}

\author{
Hong Wang, (D) ab Peisheng Zhang, (D)*ac Chonghua Zhang, ${ }^{a}$ Shu Chen, ${ }^{a}$ \\ Rongjin Zeng, ${ }^{a}$ Jiaxi Cui (D) ${ }^{\text {bd }}$ and Jian Chen (D) *ae
}

\begin{abstract}
Developing a versatile probe for targeting the lysosomes of specific cancer cells and subsequently detecting glutathione (GSH) levels is critical in disclosing the roles of GSH in the lysosomal oxidative stress of cancer cells. Herein, we demonstrate an efficient strategy for the preparation of a dual-targeting (both cancer cell- and lysosome-targeting) fluorescence nanoprobe (DTFN) that enables the imaging of GSH in the lysosomes of specific cancer cells. The nanoprobe (DTFN) is obtained by combining folic acid (FA)-modified photostable aggregation-induced emission dots with GSH-responsive manganese dioxide $\left(\mathrm{MnO}_{2}\right)$ nanosheets via electrostatic interactions. DTFN has outstanding characteristics of good water dispersity, delightful photostability, shorter responsive time $(\sim 5 \mathrm{~min})$ and wide $\mathrm{pH}$-response range. Intracellular experiments showed that the as-prepared DTFN could be preferentially internalized into a folate receptor (FR)-positive cancer cells via the FR-mediated endocytosis. Subsequently, with the aid of the positively charged amino moiety of the nanoprobe, DTFN can selectively accumulate in lysosomes and successfully achieve the real-time imaging of the lysosomal GSH levels in FR-positive cancer cells. This study highlights a strategy to design a versatile dual-targeting fluorescence probe for enhanced cancer imaging.
\end{abstract}

\section{Introduction}

Cancer, as a destructive and prevalent disease worldwide, seriously affects human health and arouses widespread concern. As important acidic digestive organelles of the endocytic and autophagic pathways in cancer cells, lysosomes are a crucial target of uncontrolled oxidative processes to free radical damage. ${ }^{1,2}$ For lysosomal oxidative stress, glutathione (GSH) as

\footnotetext{
${ }^{a}$ Key Laboratory of Theoretical Organic Chemistry and Function Molecule, Ministry of Education, Hunan Provincial Key Laboratory of Controllable Preparation and Functional Application of Fine Polymers, Hunan Province College Key Laboratory of QSAR/QSPR, Hunan Provincial Key Lab of Advanced Materials for New Energy Storage and Conversion, School of Chemistry and Chemical Hunan University of Science and Technology, Xiangtan 411201, P. R. China.

E-mail:pshzhang07@gmail.com, cj0066@gmail.com

${ }^{b}$ Institute of Fundamental and Frontier Sciences, University of Electronic Science and Technology of China, Chengdu, Sichuan, 610054, China

${ }^{c}$ State Key Laboratory of Chemo/Biosensing and Chemometrics, Hunan University, Changsha 410082, China

${ }^{d}$ INM-Leibniz Institute for New Materials, Campus D2 2, 66123 Saarbrícken, Germany

${ }^{e}$ State Key Laboratory of Luminescent Materials and Devices, South China University of Technology, Guangzhou 510640, P. R. China

$\dagger$ Electronic supplementary information (ESI) available. See DOI: 10.1039/ d0ma00124d
}

an effective cellular antioxidant plays important roles in the homeostasis of oxidative stress state because it can effectively eliminate reactive oxygen species. ${ }^{3,4}$ Thus, there is an indigenous incentive to design and prepare practical tools for efficiently detecting lysosomal GSH levels within cancer cells to reveal the roles of GSH in the lysosomal oxidative stress of cancer cells.

On account of numerous advantages including non-invasive, simple preparation, high sensitivity, and high spatial resolution, fluorescence probes coupled with fluorescence microscopy imaging have become a powerful supporting tool for the detection of various analytes. ${ }^{5-25}$ So far, much effort has gone into the development of unique GSH fluorescence probes. ${ }^{26-28}$ However, most of them were either cancer cells- or subcellular organellespecific targeting GSH fluorescence probes; ${ }^{29-38}$ however, single targeting systems were still unable to achieve efficient GSH detection. It is known that to realize the goal of efficiently detecting GSH in lysosome of specific cancer cells, an ideal GSH fluorescence probe should be constructed by combining specific cancer cell- and lysosome-targeting functions together with a GSH probe.

For the dual-targeting (both cancer cell- and lysosometargeting) GSH fluorescence probe system, the one key challenge is to integrate the targeting ligand, fluorophore, GSH-responsive group and other functional groups into one system. In general, 
because of the limited number of attachment sites, it is difficult to incorporate effectively multiple functional groups in one small organic molecule-based fluorophore. Alternatively, polymeric nanoparticles formed by amphiphilic diblock copolymers are promising candidates for constructing a dual-targeting GSH fluorescence probe due to the flexible structural design, suitable water solubility, favorable biocompatibility and so on. ${ }^{39-46}$ Up to now, several polymeric nanoparticle-based dual-targeting nanoprobes have been developed for cancer imaging and therapy. ${ }^{4-54}$ However, to the best of our knowledge, dualtargeting fluorescence polymeric nanoprobes for efficiently detecting lysosomal GSH within specific cancer cells have not yet been reported.

Hence, a simple and versatile dual-targeting fluorescence nanoprobe (DTFN) is developed for efficiently detecting lysosomal GSH in specific cancer cells, as depicted in Scheme 1. As for this system, the folic acid (FA)-modified photostable aggregationinduced emission (AIE) dots were first synthesized via acoprecipitation strategy.

As the nanocarrier, the amphiphilic block copolymers show multiple purposes: (1) the incorporation of polyethylene glycol chains could not only possess good water dispersibility and excellent biocompatibility but also enhance the photostability of AIE fluorogen for a long time; (2) the efficient introduction of a cancer targeting unit (folic acid, FA) into nanoprobe to enhance the effective uptake of the folate receptor (FR) overexpressed cancer cells and efficiently reduce the nonspecific uptake by normal cells; ${ }^{47,55-57}$ (3) the positively charged amino moiety enables the nanoparticles enter into lysosomes. ${ }^{45,58}$ Subsequently, GSH-responsive manganese dioxide $\left(\mathrm{MnO}_{2}\right)$ nanosheets, which own the ability to quench the fluorescence of the AIE fluorogen, are absorbed onto the surface of AIE dots based on electrostatic interaction to obtain DTFN. Our results suggest

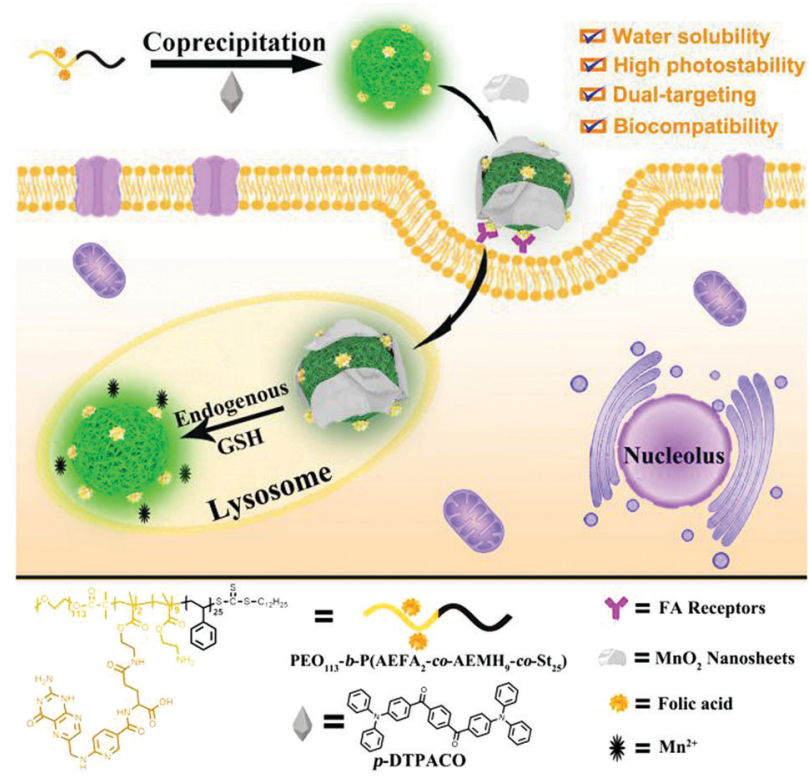

Scheme 1 Schematic illustration of dual-targeting fluorescence nanoprobe (DTFN) for efficiently detecting lysosomal GSH within cancer cells. that the dual-targeting strategy could allow DTFN to selectively internalize into the lysosomes of FR-positive cancer cells and successfully achieve efficient GSH detection.

\section{Experimental section}

\section{Materials and apparatus}

All the solvents and chemical materials mentioned in this study were purchased and used directly. 1,4-Phenylenebis((4(diphenylamino)phen-yl)methanone) ( $p$-DTPACO) and the PEO macro-RAFT agent $\left(\mathrm{PEO}_{113}\right.$-TTC) were prepared via a procedure reported in our previous study. ${ }^{45,59} \mathrm{MnO}_{2}$ nanosheets were synthesized according to the reported methods. ${ }^{60}$

\section{Results and discussion}

Scheme 1 illustrated the design strategy of DTFN for the efficient lysosomal GSH detection within specific cancer cells. First, the amphiphilic block copolymer $\mathrm{PEO}_{113}-b$-P(AEFA 2 -co$\mathrm{AEMH}_{9}-\mathrm{CO}-\mathrm{St}_{25}$ ) containing both folic acid (FA) and amino group was synthesized through the reversible addition fragmentation chain transfer (RAFT) polymerization and the further chemical graft reactions (Scheme S1, ESI $\dagger$ ). The related intermediates were confirmed via ${ }^{1} \mathrm{H}$ NMR, GPC, and absorption spectra (Fig. S1-S4 and Table S1, ESI $\dagger$ ).

Subsequently, the FA-modified AIE dots with positive charge (+11.2 mV) were synthesized using $\mathrm{PEO}_{113}-b-\mathrm{P}\left(\mathrm{AEFA}_{2}-\mathrm{co}-\mathrm{AEMH}_{9}-\mathrm{co}-\right.$ $\left.\mathrm{St}_{25}\right)$ and $p$-DTPACO via a co-precipitation strategy. ${ }^{45,59}$ The data based on the transmission electron microscopy (TEM) and dynamic light scattering (DLS) showed that the morphology of FA-modified AIE dots was spherical and the average size was about $40 \mathrm{~nm}$ (Fig. S5A and S6, ESI $\dagger$ ). Finally, the as-prepared $\mathrm{MnO}_{2}$ nanosheets with negative charge were loaded on the surface of the positively charged FA-modified AIE dots via electrostatic interaction to obtain DTFN (Fig. S7, ESI $\dagger$ ). ${ }^{60}$ The TEM data also confirmed the successful synthesis of DTFN (Fig. S5B, ESI $\dagger$ ). For the FA-modified AIE dots and DTFN, the obvious band at $1630 \mathrm{~cm}^{-1}$ of the amine group $\left(-\mathrm{NH}_{2}\right)$ can be seen in Fig. S8 (ESI $\dagger$ ), indicating that they contained the positively charged amino moiety $\left(-\mathrm{NH}_{2}\right)$. In addition, compared with the FA-modified AIE dots, the band (Mn-O) at $610 \mathrm{~cm}^{-1}$ of DTFN obviously varied (Fig. S8, ESI $\dagger$ ). The above results clearly demonstrated that the $\mathrm{MnO}_{2}$ nanosheets were loaded on the surface of the FA-modified AIE dots to obtain DTFN.

Interestingly, the fluorescence emission of the FA-modified AIE dots overlapped with the absorbance spectra of the $\mathrm{MnO}_{2}$ nanosheets (Fig. S9, ESI $\dagger$ ), leading to the occurrence of the fluorescence resonance energy transfer (FRET) effect from AIE dots to the $\mathrm{MnO}_{2}$ nanosheets. ${ }^{60,61}$ Thus, an obvious quenched fluorescence can be visualized upon the addition of $\mathrm{MnO}_{2}$ nanosheets to the solution of FA-modified AIE dots. In addition, a maximum fluorescence quenching degree of FA-modified AIE dots up to $78 \%$ can be observed upon the addition of $300 \mu \mathrm{g} \mathrm{mL}$ $\mathrm{MnO}_{2}$ nanosheets (Fig. S10, ESI $\dagger$ ).

First, the fluorescence titration of DTFN to GSH was performed in a PBS buffered solution ( $\mathrm{pH}$ 7.4). The nanoprobe DTFN itself 

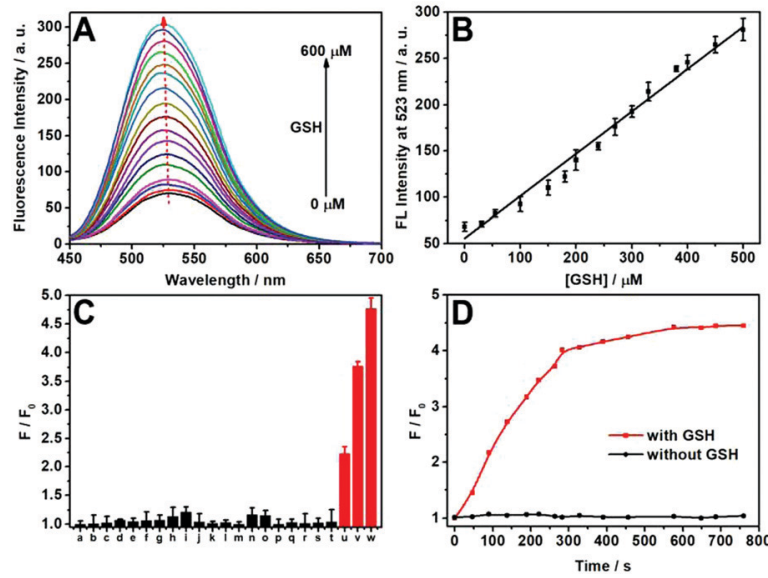

Fig. 1 (A) Fluorescence titration spectra of DTFN $\left(0.3 \mathrm{mg} \mathrm{mL}^{-1}\right)$ upon the addition of $\mathrm{GSH}(0-600 \mu \mathrm{M})$. (B) The linear relationship between the fluorescence intensity $\left(/_{523}\right)$ and GSH $(0-500 \mu \mathrm{M})$. (C) Fluorescence intensity ratio $\left(F / F_{0}\right)$ of DTFN upon exposure to various potential interferences, (a) blank (b) $\mathrm{Na}^{+}(2 \mathrm{mM}),(\mathrm{c}) \mathrm{K}^{+}(2 \mathrm{mM}),(\mathrm{d}) \mathrm{Co}^{2+}(2 \mathrm{mM}),(\mathrm{e}) \mathrm{Ca}^{2+}$ $(2 \mathrm{mM}),(\mathrm{f}) \mathrm{Mg}^{2+}(2 \mathrm{mM}),(\mathrm{g}) \mathrm{Ni}^{2+}(2 \mathrm{mM}),(\mathrm{h}) \mathrm{Cu}^{2+}(2 \mathrm{mM}),(\mathrm{i}) \mathrm{Fe}^{3+}(2 \mathrm{mM})$, (j) Ser (2 mM), (k) Asn (2 mM), (l) Thr (2 mM), (m) Gln (2 mM), (n) Gly (2 mM), (o) Ala (2 mM), (p) Arg (2 mM), (q) His (2 mM), (r) Met (2 mM), (s) BSA (1 mg mL $\left.{ }^{-1}\right)$, (t) glucose $(2 \mathrm{mM})$, (u) GSH $(200 \mu \mathrm{M})$, (v) GSH $(400 \mu \mathrm{M})$, (w) GSH $(600 \mu M)$. (D) Time-dependent fluorescent intensity ratio $\left(F / F_{0}\right)$ of DTFN in the absence of GSH (black) and in the presence of GSH (600 $\mu \mathrm{M})$ (red). Note: $F_{0}$ and $F$ are the fluorescence intensity of DTFN without and with addition of GSH, respectively.

displayed a weak fluorescence owing to the occurrence of the FRET effect from AIE dots to $\mathrm{MnO}_{2}$ nanosheets. However, the $\mathrm{MnO}_{2}$ nanosheets reduced to $\mathrm{Mn}^{2+}$ with the addition of $\mathrm{GSH}$, and thus inhibit the FRET process, resulting in the recovery of green fluorescence (Fig. 1A). In addition, a class linear relationship $\left(R^{2}=0.9855\right)$ can be observed by plotting $I_{523}$ and GSH concentration (0-500 $\mu \mathrm{M})$ (Fig. 1B) and detection limit (LOD) was achieved down to $1.03 \mu \mathrm{M}(\mathrm{S} / \mathrm{N}=3)$ (Fig. S11, ESI $\dagger$ ). Moreover, the photos of DTFN towards different GSH concentrations were observed using visible light and UV light radiation. As depicted in Fig. S12 (ESI $\dagger$ ), with the increase in the GSH concentration $(0-600 \mu \mathrm{M})$, the fluorescence of DTFN revealed an obvious increase, accompanying with the obvious color change of DTFN from yellow to colorless.

Subsequently, the selectivity of DTFN for GSH over various other analytes was carried out. As depicted in Fig. 1C, no significant change in the emission ratio $\left(F / F_{0}\right)$ can be visualized when DTFN was treated with other analytes including ions $\left(\mathrm{Na}^{+}, \mathrm{K}^{+}, \mathrm{Co}^{2+}, \mathrm{Ca}^{2+}, \mathrm{Mg}^{2+}, \mathrm{Ni}^{2+}, \mathrm{Cu}^{2+}\right.$, and $\left.\mathrm{Fe}^{3+}\right)$, amino acids (Ser, Asn, Thr, Gln, Gly, Ala, Arg, His, and Met), BSA and glucose. However, GSH can trigger a large enhancement in the emission ratio $\left(F / F_{0}\right)$. Notably, various literatures indicated that the content of GSH in cancer cells was about 1000-fold more than that of cysteine (Cys) or homocysteine (Hcy). ${ }^{62-64}$ In view of these reports, a contrast experiment on the fluorescence intensity ratio $\left(F / F_{0}\right)$ was conducted between $\mathrm{GSH}$, Cys and Hcy. As exhibited in Fig. S13 (ESI $\dagger$ ), both Cys and Hcy revealed tiny effects on the detection of GSH even their concentration $(60 \mu \mathrm{M})$ was as high as one tenth relative to GSH $(600 \mu \mathrm{M})$.
These results demonstrated the good selectivity of DTFN towards GSH against other various analytes. In addition, the response time of DTFN towards GSH was less than $5 \mathrm{~min}$, which is benefitial for the imaging of endogenous GSH in live cells.

Next, the $\mathrm{pH}$-dependent fluorescent intensity $\left(I_{523}\right)$ of DTFN with and without GSH was also explored (Fig. S14, ESI $\dagger$ ). Without GSH, a relatively weakened fluorescence intensity $\left(I_{523}\right)$ can be observed at the pH range from 4.0 to 9.0. However, a significant fluorescence enhancement of DTFN with GSH was observed in a wide $\mathrm{pH}$ range from 4.0 to 9.0, indicating that DTFN was an excellent tool for sensing GSH in the physiological environment. The photostability of DTFN without and with GSH was also conducted under the irradiation of UV light

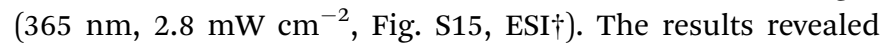
that the emission intensity $\left(I_{523}\right)$ of DTFN with GSH revealed only a slight change $(<5 \%)$ upon UV irradiation for $90 \mathrm{~min}$, indicating that DTFN had excellent photostability. In addition, to investigate the practicability of DTFN, the adscititious GSH contents in the blood serum sample were detected by the DTFN probe. As exhibited in Table S2 (ESI $\dagger$ ), the alter RSD was less than $0.55 \%$, and the recoveries revealed a change in the range from $90 \%$ to $103 \%$. The result illustrated that DTFN can serve as an excellent fluorescent nanoprobe for GSH detection in blood serum.

Based on the above admirable features of DTFN to detect GSH, the ability of DTFN to image endogenous GSH in the lysosomes of FR-positive cancer cells was further evaluated. Before imaging, standard MTT assays were utilized to appraise the cytotoxicity of DTFN, as presented in Fig. S16 (ESI $\dagger$ ). The results indicated that DTFN had low cytotoxicity towards HeLa cells.

First, to confirm the cellular targeting ability of DTFN, FA-positive HeLa cells and FA-negative A549 cells were selected and co-cultured with DTFN, respectively. As can be seen from Fig. 2, the HeLa cells displayed stronger green fluorescence than A549 cells, demonstrating that DTFN had excellent targeting ability for FR-positive cells. It has been reported that the specific uptake of FA-modified nanoparticles by HeLa cells was proceeded via a FA-receptor-mediated endocytic process. ${ }^{47,55-57}$

To further confirm this cellular uptake mechanism, the HeLa cells were incubated with DTFN in FA-containing and FA-free culture medium, respectively (Fig. S17, ESI $\dagger$ ). When the HeLa cells were treated with DTFN in the FA-free culture medium, a bright green fluorescence was seen, whereas the HeLa cells treated with DTFN in the FA-containing culture medium depicted a weak green fluorescence. The results confirmed the important role of FA in guiding DTFN to FR-positive cells.

It has been well-documented that nanoprobes often accumulated in lysosomes via the interplay of size and surface charge: (1) nanostructure could be taken up by cells by the energy-dependent endocytosis pathway whose destination is lysosomes; ${ }^{65,66}$ (2) the positively charged amino moiety enables the nanoparticles to enter into lysosomes. ${ }^{45,58,67,68}$ To inspect the subcellular targeting ability of DTFN, HeLa cells pre-treated with DTFN were co-incubated with Lyso-Tracker Red and MitoTracker Red, respectively. As displayed in Fig. 3, the green fluorescence of DTFN merged well with the red fluorescence 


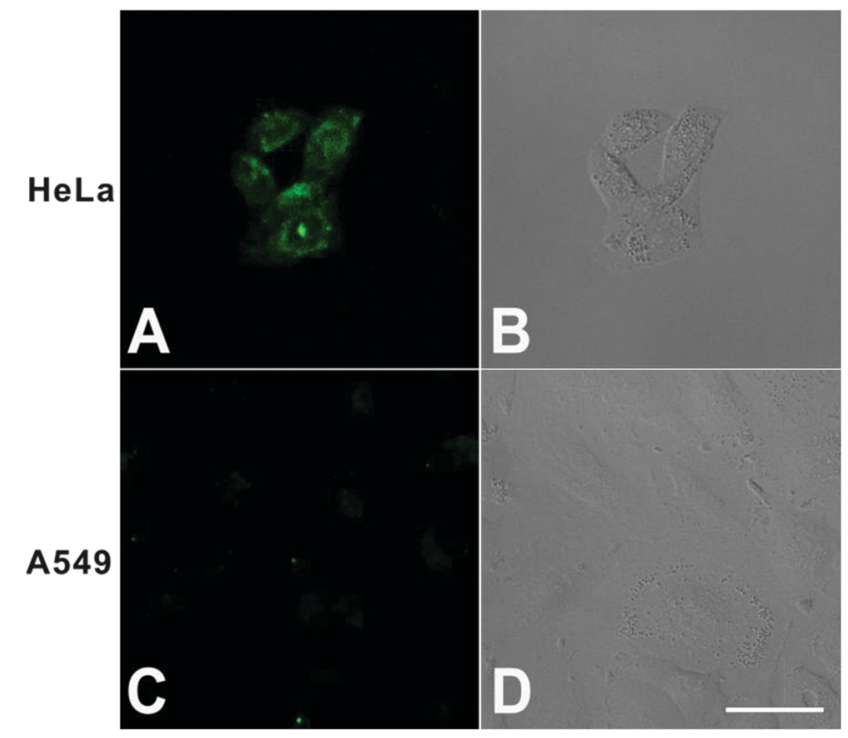

Fig. 2 Confocal fluorescence microscopy images of HeLa (A and B) and A549 cells ( $C$ and D) treated with DTFN $\left(0.12 \mathrm{mg} \mathrm{mL}^{-1}\right)$. Scale bar: $40 \mu \mathrm{m}$.

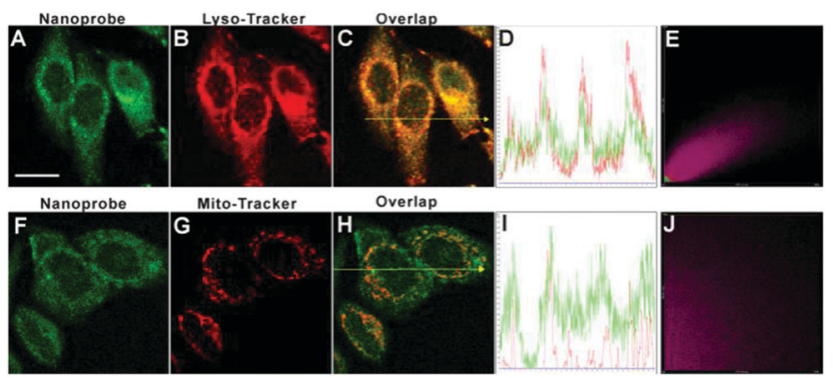

Fig. 3 Confocal fluorescence images of DTFN-loaded HeLa cells incubated with either Lyso-Tracker Red or Mito-Tracker Red. (A) and (F): green channel for DTFN ( $\lambda_{\mathrm{ex}}=488 \mathrm{~nm}, \lambda_{\mathrm{em}}=500-530 \mathrm{~nm}$ ). (B) and (G): red channel for Lyso-Tracker Red and Mito-Tracker. Red ( $\lambda_{\mathrm{ex}}=543 \mathrm{~nm}, \lambda_{\mathrm{em}}=$ 560-610 nm). (C) Merged image of (A) and (B). (D) Intensity profile of regions of interest (ROI) across HeLa cells as indicated by the yellow arrow in (C). (E) Correlation plot of Lyso-Tracker Red and DTFN intensities. (H) Merged image of (F) and (G). (I) Intensity profile of the regions of interest (ROI) across HeLa cells, as indicated by the yellow arrow in $(\mathrm{H})$. (J) Correlation plot of Mito-Tracker Red and DTFN intensities. Scale bar: $20 \mu \mathrm{m}$.

of Lyso-Tracker Red (Pearson's correlation coefficient: 0.89). In contrast, the poor colocalization with Mito-Tracker Red (Pearson's correlation coefficient: 0.32) was observed. The above results (High Pearson's correlation coefficient) clearly demonstrated that DTFN can specially accumulate in the lysosomes via the interplay of surface charge and size.

Next, we sought to evaluate the feasibility of DTFN for realtime monitoring endogenous GSH in the lysosomes of HeLa cells (Fig. 4). An obvious green fluorescence enhancement can be visualized with increasing incubation time (Fig. 4B-E), suggesting that the high capacity of DTFN for imaging endogenous GSH in real time. To confirm the specificity of DTFN for intracellular GSH, the HeLa cells were pretreated with the effective GSH inhibitor ( $N$-ethylmaleimide, NEM) and then
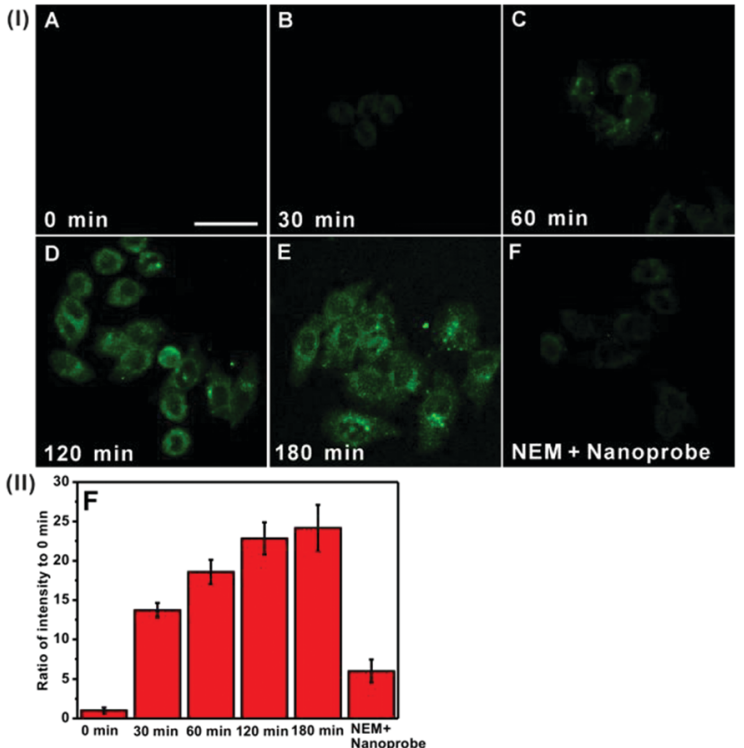

Fig. 4 (I) Confocal images of HeLa cells stained with the nanoprobe (DTFN, $0.12 \mathrm{mg} \mathrm{mL}^{-1}$ ) for 0 (A), 30 (B), 60 (C), 120 (D), $180 \mathrm{~min}$ (E). (F) Confocal images of NEM-pretreated HeLa cells stained with the nanoprobe (DTFN) for $180 \mathrm{~min}$. (II) Quantification of relative fluorescence intensity using Image $\mathrm{J}$ software. Values are the mean $\pm \mathrm{SD}(n=5)$. Scale bar: $20 \mu \mathrm{m}$

incubated with DTFN (Fig. 4F). The green fluorescence of cells was remarkably suppressed, confirming that the green fluorescence enhancement of HeLa cells was indeed induced by the endogenous GSH. Apparently, the above results verified that DTFN can be served as an excellent tool to selectively monitor endogenous GSH in the lysosomes of HeLa cells.

\section{Conclusions}

In summary, a novel dual-targeting fluorescence nanoprobe (DTFN) was rationally synthesized, and we have demonstrated its excellent capability for efficiently detecting endogenous GSH in the lysosomes of FR-positive HeLa cells. This nanoprobe displayed several advantages over some of the previously reported GSH probes (Table S3, ESI $\dagger$ ): (1) the utilization of photostable AIE fluorogen can effectively avoid the aggregationcaused quenching (ACQ) phenomenon of traditional organic small molecule-based GSH probes; (2) the introduction of PEG chains could greatly enhance the water dispersibility and biocompatibility of nanoprobes, while most of the traditional organic small molecule-based GSH probes are usually carried out in organic-aqueous media; (3) by virtue of the dual-targeting strategy, the nanoprobe (DTFN) can successfully achieve the efficient GSH detection in the lysosome of cancer cells. To the best of our knowledge, this is the first study to detect intracellular GSH through a dual-targeting nanoprobe. This methodology provided a new route to the rational design of novel dual-targeting nanoprobes for efficient biomarkers detection in the subcellular organelle of cancer cells. 


\section{Conflicts of interest}

There are no conflicts to declare.

\section{Acknowledgements}

We gratefully acknowledge the financial support by NSFC (51603067, 51773056, 51873058, 21705040), Hunan Provincial Natural Science Foundation of China (2020JJ3021, 2018JJ3143), China Postdoctoral Science Foundation (2018T110824), Scientific Research Fund of Hunan Provincial Education Department (Project no. 19B204), Open Project Program of State Key Laboratory of Chemo/Biosensing and Chemometrics (2018011), Open Fund of Hunan Provincial Key Laboratory of Advanced Materials for New Energy Storage and Conversion (2018TP1037-202003) and the Open Fund of the State Key Laboratory of Luminescent Materials and Devices (South China University of Technology, 2019-skllmd-09).

\section{Notes and references}

1 X. Zhang, J. Wang, X. Li and D. Wang, Cancer Lett., 2018, 439, 39-46.

2 P. Saftig and A. Haas, Nat. Cell Biol., 2016, 18, 1025-1027.

3 J. M. Estrela, A. Ortega and E. Obrador, Crit. Rev. Clin. Lab. Sci., 2008, 43, 143-181.

4 G. Pani, T. Galeotti and P. Chiarugi, Cancer Metastasis Rev., 2010, 29, 351-378.

5 T. Li, C. Yin, J. Chao, W. Zhang and F. Huo, Sens. Actuators, B, 2020, 304, 127431.

6 L. Wu, A. C. Sedgwick, X. Sun, S. D. Bull, X.-P. He and T. D. James,, Acc. Chem. Res., 2019, 529, 2582-2597.

7 S. Singha, Y. W. Jun, S. Sarkar and K. H. Ahn, Acc. Chem. Res., 2019, 52, 2571-2581.

8 Y. Yue, F. Huo, X. Pei, Y. Wang and C. Yin, Anal. Chem., 2020, 92, 6598-6603.

9 J. Zhang, X. Chai, X. P. He, H. J. Kim, J. Yoon and H. Tian, Chem. Soc. Rev., 2018, 48, 683-722.

10 T. Zhou, Y. Yang, K. Zhou, M. Jin, M. Han, W. Li and C. Yin, Sens. Actuators, B, 2019, 301, 127116.

11 L. He, B. Dong, Y. Liu and W. Lin,, Chem. Soc. Rev., 2016, 45, 6449-6461.

12 W. Zhang, F. Huo, F. Cheng and C. Yin, J. Am. Chem. Soc., 2020, 142, 6324-6331.

13 V. S. Lin, W. Chen, M. Xian and C. J. Chang, Chem. Soc. Rev., 2015, 44, 4596-4618.

14 P. Zhang, H. Wang, Y. Hong, M. Yu, R. Zeng, Y. Long and J. Chen, Biosens. Bioelectron., 2018, 99, 318-324.

15 W. Zhang, F. Huo, Y. Yue, Y. Zhang, J. Chao, F. Cheng and C. Yin, J. Am. Chem. Soc., 2020, 142, 3262-3268.

16 S. Yang, J. Jiang, A. Zhou, Y. Zhou, W. Ye, D. S. Cao and R. Yang, Anal. Chem., 2020, 92, 7194-7199.

17 H. Li, H. Lin, W. Lv, P. Gai and F. Li, Biosens. Bioelectron., 2020, 165, 112336.

18 T. Li, C. Yin, J. Chao, W. Zhang and F. Huo, Sens. Actuators, B, 2020, 305, 127336.
19 Y. Zhou, S. Yang, J. Guo, H. Dong, K. Yin, W. T. Huang and R. Yang, Anal. Chem., 2020, 92, 5787-5794.

20 W. Zhang, F. Huo and C. Yin, Org. Lett., 2019, 21(13), 5277-5280.

21 X. Wu, W. Shi, X. Li and H. Ma, Acc. Chem. Res., 2019, 52, 1892-1904.

22 P. Zhang, H. Wang, D. Zhang, X. Zeng, R. Zeng, L. Xiao, H. Tao, Y. Long, P. Yi and J. Chen, Sens. Actuators, B, 2018, 255, 2223-2231.

23 H. Wei, R. Zeng, S. Wang, C.-H. Zhang, S. Chen, P. Zhang and J. Chen, Mater. Chem. Front., 2020, 4, 862-868.

24 Y. Yang, T. Zhou, M. Jin, K. Zhou, D. Liu, X. Li, F. Huo, W. Li and C. Yin, J. Am. Chem. Soc., 2020, 142, 1614-1620.

25 J. Ren, P. Zhang, H. Liu, C. Zhang, Y. Gao, J. Cui and J. Chen, Sens. Actuators, B, 2020, 304, 127299.

26 H. Chen, Y. Tang and W. Lin, TrAC, Trends Anal. Chem., 2016, 76, 166-181.

27 J. Liu, Y.-Q. Sun, Y. Huo, H. Zhang, L. Wang, P. Zhang, D. Song, Y. Shi and W. Guo, J. Am. Chem. Soc., 2014, 136, 574-577.

28 L.-Y. Niu, Y.-Z. Chen, H.-R. Zheng, L.-Z. Wu, C.-H. Tung and Q.-Z. Yang, Chem. Soc. Rev., 2015, 44, 6143-6160.

29 Z. Yuan, L. Gui, J. Zheng, Y. Chen, S. Qu, Y. Shen, F. Wang, M. Er, Y. Gu and H. Chen, ACS Appl. Mater. Interfaces, 2018, 10, 30994-31007.

30 M. H. Lee, J. H. Han, P.-S. Kwon, S. Bhuniya, J. Y. Kim, J. L. Sessler, C. Kang and J. S. Kim, J. Am. Chem. Soc., 2012, 134, 1316-1322.

31 R. Guo, F. Huang, B. Zhang, Y. Yan, J. Che, Y. Jin, Y. Zhuang, R. Dong, Y. Li, B. Tan, R. Song, Y. Hu, X. Dong, X. Li and N. Lin, Theranostics, 2019, 9, 3515-3525.

32 D. Feng, Y. Song, W. Shi, X. Li and H. Ma, Anal. Chem., 2013, 85, 6530-6535.

33 S. Zang, X. Kong, J. Cui, S. Su, W. Shu, J. Jing and X. Zhang, J. Mater. Chem. B, 2020, 8, 2660-2665.

34 Q. Gao, W. Zhang, B. Song, R. Zhang, W. Guo and J. Yuan, Anal. Chem., 2017, 89, 4517-4524.

35 M. Cao, H. Chen, D. Chen, Z. Xu, S. H. Liu, X. Chen and J. Yin, Chem. Commun., 2016, 52, 721-724.

36 H. Zhao, X. Wen, W. Li, Y. Li and C. Yin, J. Mater. Chem. B, 2019, 7, 2169-2176.

37 Z. Xu, X. Huang, X. Han, D. Wu, B. Zhang, Y. Tan, M. Cao, S. H. Liu, J. Yin and J. Yoon, Chemistry, 2018, 4, 1609-1628.

38 S.-Y. Lim, K.-H. Hong, D. I. Kim, H. Kwon and H.-J. Kim, J. Am. Chem. Soc., 2014, 136, 7018-7025.

39 K. Li and B. Liu, Chem. Soc. Rev., 2014, 43, 6570-6597.

40 Q. Miao, C. Xie, X. Zhen, Y. Lyu, H. Duan, X. Liu, J. V. Jokerst and K. Pu, Nat. Biotechnol., 2017, 35, 1102-1110.

41 L. Bau, P. Tecilla and F. Mancin, Nanoscale, 2011, 3, 121-133.

42 L. Feng, C. Zhu, H. Yuan, L. Liu, F. Lv and S. Wang, Chem. Soc. Rev., 2013, 42, 6620-6633.

43 X. Huang, J. Song, B. C. Yung, X. Huang, Y. Xiong and X. Chen, Chem. Soc. Rev., 2018, 47, 2873-2920.

44 J. Hu and S. Liu, Acc. Chem. Res., 2014, 47, 2084-2095.

45 H. Wang, P. Zhang, Y. Hong, B. Zhao, P. Yi and J. Chen, Polym. Chem., 2017, 8, 5795-5802. 
46 J. Yu, Y. Rong, C. T. Kuo, X. H. Zhou and D. T. Chiu, Anal. Chem., 2016, 89, 42-56.

47 J. Xu, F. Zeng, H. Wu, C. Yu and S. Wu, ACS Appl. Mater. Interfaces, 2015, 7, 9287-9296.

48 S. Chen, J. Fan, W. Qiu, F. Liu, G. Yan, X. Zeng and X. Zhang, J. Mater. Chem. B, 2018, 6, 1543-1551.

49 H. Chen, Y. Chen, H. Yang, W. Xu, M. Zhang, Y. Ma, S. Achilefu and Y. Gu, Polym. Chem., 2014, 5, 4734-4746.

50 J. Wang, Y. Yang, Y. Zhang, M. Huang, Z. Zhou, W. Luo, J. Tang, J. Wang, Q. Xiao, H. Chen, Y. Cai, X. Sun, Y. Wang and Y. Ke, Adv. Funct. Mater., 2016, 26, 7873-7885.

51 B. Y. Liu, X. Y. He, C. Xu, L. Xu, S. L. Ai, S. X. Cheng and R. X. Zhuo, Biomacromolecules, 2018, 19, 2957-2968.

52 Y. Zhong, F. Meng, C. Deng and Z. Zhong, Biomacromolecules, 2014, 15, 1955-1969.

53 S. Fu, M. Liang, Y. Wang, L. Cui, C. Gao, X. Chu, Q. Liu, Y. Feng, W. Gong, M. Yang, Z. Li, C. Yang, X. Xie, Y. Yang and C. Gao, ACS Appl. Mater. Interfaces, 2019, 11, 1841-1854.

54 L. Dai, R. Cai, M. Li, Z. Luo, Y. Yu, W. Chen, X. Shen, Y. Pei, X. Zhao and K. Cai, Chem. Mater., 2017, 29, 6976-6992.

$55 \mathrm{~J} . \mathrm{Xu}, \mathrm{F}$. Zeng, H. Wu, C. Hu and S. Wu, Biomacromolecules, 2014, 15, 4249-4259.

56 J. Fan, G. Fang, F. Zeng, X. Wang and S. Wu, Small, 2013, 9, 613-621.
57 J. Fan, F. Zeng, S. Wu and X. Wang, Biomacromolecules, 2012, 13, 4126-4137.

58 J. Chen, Y. Tang, H. Wang, P. Zhang, Y. Li and J. Jiang, J. Colloid Interface Sci., 2016, 484, 298-307.

59 W. Zhong, X. Zeng, J. Chen, Y. Hong, L. Xiao and P. Zhang, Polym. Chem., 2017, 8, 4849-4855.

60 H. M. Meng, Z. Jin, Y. Lv, C. Yang, X. B. Zhang, W. Tan and R. Q. Yu, Anal. Chem., 2014, 86, 12321-12326.

61 R. Deng, X. Xie, M. Vendrell, Y. T. Chang and X. Liu, J. Am. Chem. Soc., 2011, 133, 20168-20171.

62 F. Yu, P. Li, B. Wang and K. Han, J. Am. Chem. Soc., 2013, 135, 7674-7680.

63 Q. Wang, Y. Zhang, X. Wang, Y. Wu, C. Dong and S. Shuang, Analyst, 2019, 144, 1988-1994.

64 Q. Y. Cai, J. Li, J. Ge, L. Zhang, Y. L. Hu, Z. H. Li and L. B. Qu, Biosens. Bioelectron., 2015, 72, 31-36.

65 X. Ma, Y. Wu, S. Jin, Y. Tian, X. Zhang, Y. Zhao, L. Yu and X.-J. Liang, ACS Nano, 2011, 5, 8629-8639.

66 D. Huo, Q. Chen, J. Xue, S. Shen and Y. Xia, Adv. Mater., 2017, 29, 1703702.

67 Y. Jiang, S. Huo, T. Mizuhara, R. Das., Y. Lee, S. Hou, D. F. Moyano, B. Duncan, X.-J. Liang and V. M. Rotello, ACS Nano, 2015, 9, 9986-9993.

68 J. Mosquera, I. García and L. M. Liz-Marzán, Acc. Chem. Res., 2018, 51, 2305-2313. 